\title{
Digital Immersive Virtual Environments and Instructional Computing
}

\author{
Jim Blascovich • Andrew C. Beall
}

(C) The Author(s) 2010. This article is published with open access at Springerlink.com

\begin{abstract}
This article reviews theory and research relevant to the development of digital immersive virtual environment-based instructional computing systems. The review is organized within the context of a multidimensional model of social influence and interaction within virtual environments that models the interaction of four theoretical factors: theory of mind, communicative realism, behavioral systems level, and situational self-relevance. The ways in which social interactional processes in digital immersive virtual environments may be easily filtered and transformed in ways relevant to instructor-learner interactions are discussed. Relevant experimental investigations are reviewed that indicate their potential to increase the adaptivity of immersive virtual environment systems for learners and instructors.
\end{abstract}

Keywords Virtual reality · Instructional computing · Transformed social interactions

\section{The Subjective Nature of Reality}

Humans can perceive things that do not exist in their immediate natural environments, sometimes in ways they are motivated to perceive. Such perceptions occur endogenously via dreams and daydreams and exogenously primarily via a long history of communicative media inventions including: storytelling, painting and sculpture, theatre, manuscripts and books, photography and cinema, radio and television, and, recently, digital-immersive virtual technology.

Digital immersive virtual environment technology (IVET) is the most recent in the history of media-based technologies. This technology "transports" users via various sensory

J. Blascovich $(\bowtie) \cdot$ A. C. Beall

Research Center for Virtual Environments and Behavior, Department of Psychology, Psychology East, University of California, Santa Barbara, Santa Barbara, CA 93106, USA

e-mail: blascovi@psych.ucsb.edu

A. C. Beall

e-mail: beall@psych.ucsb.edu 
interfaces (e.g., head-mounted displays; see Fig. 1) to synthetic environments created using digital technology for various purposes such as entertainment and instruction. Compared to previous technologies, IVET increases the likelihood of psychological immersion in virtual worlds. Because IVET also allows for heretofore unlikely or even impossible ways for people to interact, it enables instructors, human or agentic, to interact with students in novel ways that promote learning.

Every media technology for which there is a historical record has been used didactically by teachers and students in ways that promote learning. To the extent that students become immersed or more attentive in such media-assisted perceptions, the assumption is that media-based social transactions will be more efficient and, thereby, more effective. In terms of instruction, such transactions involve knowledge and skills.

Here, we discuss how certain factors determine how social influence occurs in immersive virtual environments. We start by describing our own theoretical model of social influence within such environments, pointing out possible implications for the design of pedagogical agents and avatars. Next, we described unique "transformed social interactions" that digital virtual environment technology permits and that quite likely can influence learners and instructors in positive ways. The fact that we focus on the relevance

Fig. 1 Head-mounted display apparatus

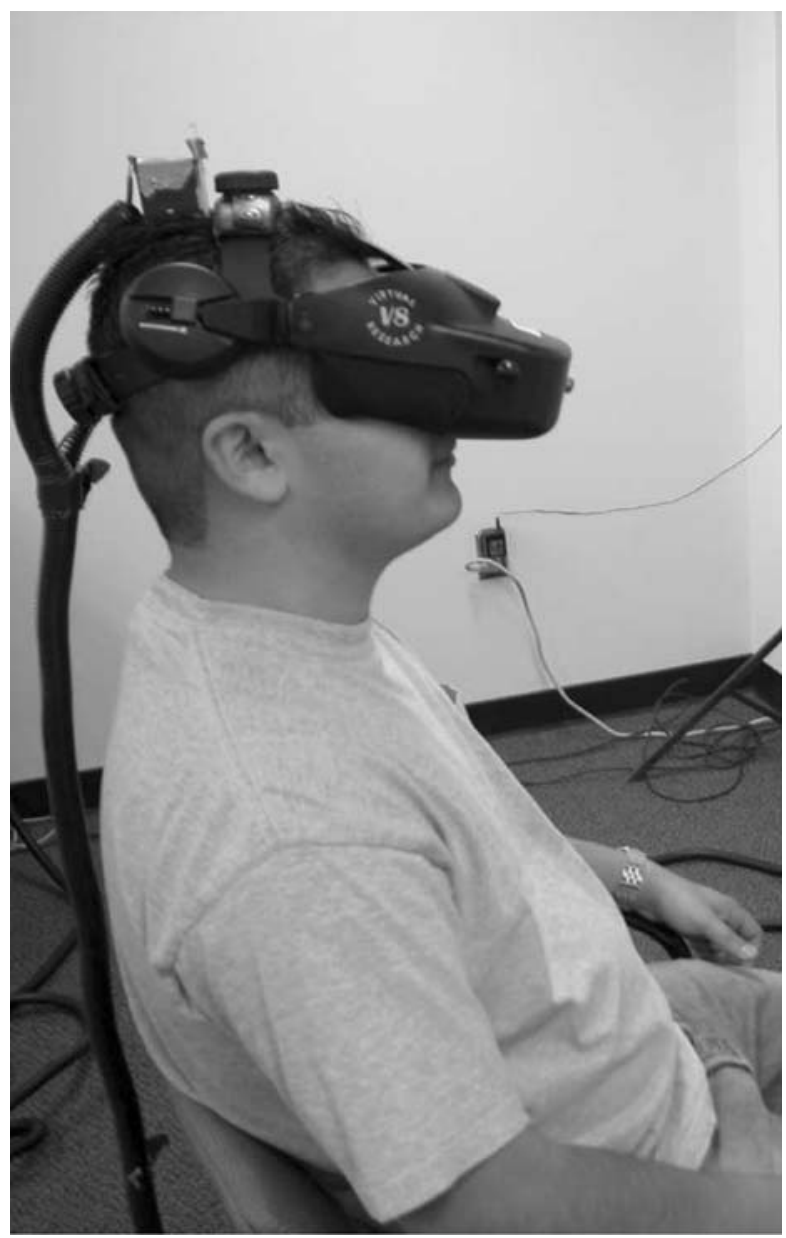


of a general theoretical social psychological model of social influence in this article is not meant to detract from other theoretical models applicable to computer instruction (see, for example, Graesser et al. 1997; Roussou et al. 2008).

\section{Social Influence and Interactions within Digital-Immersive Virtual Environments}

Social influence is important for learning in a number of respects. Instructors influence learners and vice versa. Central to the instructional process is the knowledge gained by learners. Communication is the mediating processes in instructor-learner interactions (Rummel \& Kraemer, this issue). In addition to verbal and nonverbal one-to-one communication, communication media have been adapted for the instructor-learner interaction since the beginning. One adaptation has been the creation of digital pedagogical agents to transmit instructional communication via graphical and embodied entities to learners. The literature on pedagogical agents, covered elsewhere in this issue (see Kramer $\&$ Bente, this issue), is impressive.

The latest entry on the communication media scene is digital technology. Worldwide user data reveal that digitally based human-to-human and human-to-agent interactions are ubiquitous. Billions of people use computers and a large subset (1.5 billion people at the time of this writing) of those that interact with others via the internet (Internet World Stats 2008). At recent count, over 150 million websites provide information and services of every conceivable type, including instructional ones, to people wherever and whenever they are connected. Furthermore, the relatively recent availability of so-called social networking sites facilitate social interactions asynchronously (e.g., FaceBook $($, MySpace $($ ) as well as synchronously (e.g., Second Life(C). The latter situate individuals in so-called virtual worlds in real time. In the relatively near future, we believe that immersive digital virtual environment technology, one that permits face-to-face interaction in three-dimensional digital worlds via agents and/or avatars, will likely impact education in important ways. Consequently, it is necessary to determine how social influence operates via this technology if we are to develop first-rate instructional applications.

Fundamental to understanding why and how people immerse themselves in virtual environments is familiarity with social psychological principles of social interaction, particularly with social influence processes. The term, "social influence," refers to the effects that people have on each other. Social influence effects take many forms, several of which relate directly to education including persuasion, performance facilitation or inhibition, modeling, mimicry, and memory, to name a relative few (Blascovich et al. 2002).

Over the last decade, social psychologists, computer scientists, and communication media experts have empirically examined social influence and interaction within digital immersive virtual environments (IVEs) that have implications for the development of virtual immersive education scenarios. Blascovich and colleagues (Blascovich et al. 2002) developed and refined a four-factor theoretical model of social influence and interaction in IVEs. These factors include: theory of mind, communicative realism, behavioral response system, and self-relevance.

Theory of mind

Theory of mind refers to humans' attributions of others' mental states (Premack and Woodruff 1978). The most fundamental or superordinate of these attributions involves sentience; more specifically, the attribution of sentience or non-sentience or somewhere in 
between (Garau et al. 2005). Assuming sentience, further delineation and attributions of mental states - beliefs, intentions, attitudes, motivations, knowledge, and personality, among them - set the stage for interactions among people. Persons seeing human forms walking toward them (or when they walk toward human forms) in the physical world, assume sentience and often additional mental states of those humans, including cognitive, motivational, and affective aspects; that is, a simple theory of their minds. Typically, in the physical environment, humans are not consciously aware of making these attributions.

Modulating factors for social interaction within virtual environments include the beliefs that people hold about human representations in those environments. A representation in a virtual world that is believed by others to represent an actual person is labeled an "avatar," and one believed to represent a fictional or synthetic person is labeled an "agent" (Bailenson and Blascovich 2005). Furthermore, this distinction need not be dichotomous, as digital representations can exhibit extra human powers, which some have labeled "cyborgs" (i.e., digitally enhanced representations of actual people).

It is important to note that the agent-avatar distinction is not limited to digital representations. Hence, the printed description of a protagonist in a novel; e.g., "Harry Potter," is essentially an agent as Harry Potter is fictional. In contrast, the printed name of the target character in a biography, e.g., "Winston Churchill," is essentially an avatar. Clearly, many readers react cognitively and emotionally to characters in novels and biographies based on the printed information about them as they read. Similar processes are at work when people experience theatre, film, radio and television, etc.

In IVEs, people similarly interact with digital human representations. If a person believes the digital representation is an avatar, he or she will likely act somewhat differently than if he or she believes the digital representation is an agent at least on the deliberative or conscious level. Such beliefs are also important in the physical world where people sometimes treat actual people as though they are not sentient; for example, e.g., waiters and waitresses on occasion (Goffman 1961). There is no reason to believe it is any different in virtual reality. What matters is the interplay of some additional factors.

Communicative realism

How theory of mind is informed is the focus of much scholarship and research. One important factor is the communication of animacy. Communicative realism refers to the recognizable signal quality of communicative acts. In our conception, it does not necessarily refer to the appearance of his or her avatar. Indeed, as is more obviously the case with digital agents, it refers to the realism of the apparent communicative act.

Empirically, communicative realism is a latent variable, and, therefore, by definition cannot be measured directly. However, in the Blascovich et al. (2002) theoretical model, communicative realism is regarded as a function of three related manifest variables, ones that can be measured and manipulated directly. These manifest variables, in decreasing order of importance, are: movement, anthropometric, and photographic realism.

Movement realism has played the major visual role underlying communicative realism and, therefore, attribution of sentience to digital representations in IVEs. Perceptions of human-like movements appear to arise via the activation of mirror neurons and the social brain network as Wheatley et al. (2007) have demonstrated. Movement realism need only include gross spatial translational movements in two dimensions as Heider and Simmel (1944) demonstrated long ago. However, movement realism can be enhanced via more articulated movements such as walking, gestural ones such as head nods, and micromovements such as those involved in eye gaze and facial expressions. 
Anthropometric realism is important to the extent that it supports human movement realism because certain human-like movements require certain recognizable human body parts. One cannot represent a wave without an arm and hand, a gaze without eyes, a smile without some sort of mouth, etc. Photographic realism functions only to communicate specific identity. Animators have realized for more than a century, at least implicitly, the roles these manifest movement realism variables play in immersing their audiences in the novel worlds they create.

\section{Theory of mind $\times$ communicative realism}

Figure 2 depicts the relationship between theory of mind and communicative realism in the model. As a user's theory of mind (i.e., attributions) about a digital human representation in a IVE increases toward sentience (i.e., that the representation is an avatar) and as communicative realism of the representation increases in ways appropriate to human interaction, the user experiences social verification, or the belief that he or she is enmeshed in an interaction with another (depicted by the diagonal function indicated in Fig. 2).

At some point on the increasing social verification function, a threshold is crossed, and social influence effects will occur (depicted by the indicated function that appears orthogonal to the social verification function). The functions as depicted reflect the model's predictions that social influence will occur if either theory of mind or communicative realism are relatively high. More specifically, if a user's theory of mind regarding a digital human representation is that it is an agent (i.e., represents only a computer algorithm), then near perfect communicative realism is necessary for highly self relevant and meaningful social influence to occur. If a user's theory of mind regarding a digital human representation is that it is an avatar (i.e., represents an actual human), then relatively little communicative realism is necessary for highly self-relevant meaningful social influence to occur.

The main implication of the interaction of these two factors for instructional computing systems based in digital immersive virtual environments is that the apparent communicative realism of agents, especially regarding nonverbals, must generally be much greater than that of avatars to produce comparable learning effects that are a product of social influence to occur. However, one must take into account at least two more important variables.

Behavioral response systems level

To this point, the interaction of communicative realism and theory of mind within the context of social influence within virtual worlds have been described. Two additional

Fig. 2 Partial depiction of threshold model of social influence in IVE

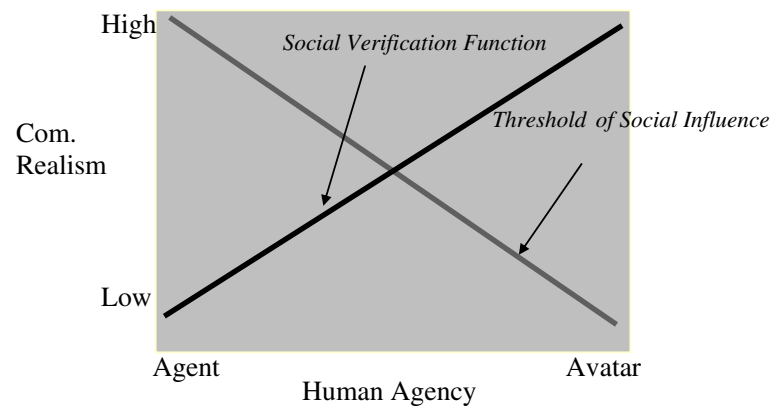


factors need be considered. For one, behavioral response systems level must be taken into account. More specifically, social influence can occur reflexively or unconsciously not only in the physical world but also in virtual worlds. Indeed, reflexive and unconscious social influence appears to be identical in both. If a human representation known to be an agent unexpectedly throws a virtual punch, the user will still exhibit a "startle" response, no matter what its level of communicative realism, of about the same magnitude as when such a punch is thrown by an avatar. Similarly, an agent's head nod is likely to elicit a mimicry response on the part of the human interactant. In early studies of movement realism (Bailenson et al. 2001), we found that participants' attribution of sentience was unnecessary to create automatic proxemic behaviors (i.e., maintenance of appropriate interpersonal distance) when they interacted with known agents. Important here is that for low level (i.e., automatic, reflexive, or unconscious) response systems agency appears irrelevant.

Much evidence indicates that people process information unconsciously and consciously simultaneously (Uleman and Bargh 1989). Furthermore, unconscious and conscious levels of mental processing can influence one another. For example, mirror neurons are active when a person consciously or unconsciously moves, when a person observes movements by another or when the person imagines herself or another person moving. People's reactions to movements of others apparently involve the same central neural pathways involved in their own movements. Such neural processing of communicative movements appears to prime users to acknowledge unconsciously that when a digital human representation, whether avatar or agent, moves like a person, it must be a person.

The implications of response level considerations for developing effective digital immersive virtual learning scenarios is clear. With regard to learning scenarios, communicative realism in terms of nonverbals is quite important especially if they are didactic agent-based.

\section{Self-relevance}

Many social interaction scenarios are highly self-relevant; that is, they are important to the goals of the people involved. For example, falling in love is undoubtedly highly selfrelevant social situation in most cultures. In contrast, chatting about the weather is probably the low end of the self-relevance. In the model, self-relevance moderates the influence of communicative realism on social influence. If situational self-relevance is high, then communicative realism must be high for people to be socially influenced by agents at the conscious or deliberative level. If situational self-relevance is low, then communicative realism need not be high for such social influence to occur.

Clearly, instructional or learning contexts and scenarios are often quite goal-relevant and, hence, self-relevant to individuals. Self-relevance, therefore, needs to be taken into account when digital immersive virtual environmental instructional systems are developed. If they are based on instructional agents special care must be taken to ensure the communicative realism of the agents.

\section{Transformed Social Interactions}

In the past, synchronous communicative media technology (e.g., telephone, video conferencing) presented high fidelity audio and video signals to individuals. Little, if anything, could be done to alter such signals on-the-fly during social interactions except for filtering signal quality (e.g., disguising one's voice or image). In contrast, IVET permits 
much more in the way of filtering and altering signals, especially visual ones, on-the-fly during immersive social interactions. Given such technical capabilities and what is known from the long history of scholarly work on nonverbal behaviors and social influence, IVE developers should be able to imbue IVE users (e.g., instructors and students) with extraordinary social influence powers thereby transforming the quality of social interaction and social influence within IVEs. Recognizing this possibility, we (Bailenson 2006; Bailenson and Beall 2006; Bailenson et al. 2005) described a theoretical framework, in which three major types of transformed social interactions (TSIs) were delineated.

One type involves transformations of self-presentation. This category of TSIs involves systematically altering the appearance and/or behaviors of avatars from those of the participant being tracked. For example, an avatar can appear younger or older than the person it represents. Or, an avatar can be made to walk forward when the person it represents walks backwards. Furthermore, a person's avatar can be tailored to the other interactants such that it appears younger to one interactant and older to another, or its movements can differ uniquely for each other interactant.

A second type of TSI involves transformations of situational context. This category of TSIs involves systematically altering the spatial or temporal aspects of a social interaction. For example, from each person's point of view, everyone in a IVE-based conference can find themselves (via their avatar) sitting at the head of the conference table with the others appearing to sit elsewhere. Because IVET episodes can be easily recorded, individual participants may be able to rewind the social interaction to verify some bit of past conversation and "fast forward" at somewhat faster than normal speed to catch back up with the ongoing interaction.

A third type of TSI involves transformations of sensory abilities. Such TSIs provide extrasensory tools to interactants within IVEs. For example, an individual can literally take the visual view of other interactants, watching through their avatars" "eyes" and seeing what they see as the other interactants change their gaze. They can also receive data via the IVET indicating the movements of other participants.

Finally, although a discussion of ethics is not within the scope of this article, the notion of TSIs certainly raises many issues. Though technologically feasible, TSIs raise the issue of dual use (i.e., "for moral or immoral purposes?") which has plagued scientific and technological advances for millennia.

\section{TSIs and Instructional Computing}

All three types of TSIs can be employed in instructional IVEs. Indeed, the possible combinations of transformations of self-presentation, context, and sensory abilities are seemingly endless. The question is whether or not they can be used to improve instruction and learning. Here, we review some initial studies.

\section{Self-presentation TSI: non-zero sum gaze}

Eye gaze appears to be one of the most powerful nonverbal signals available to people. Gaze increases social influence in almost any context and increases the persuasive powers of presenters (Burgoon et al. 2002). In the natural world, mutual or shared gaze is limited to a single pair of individuals at any one time. However, in IVEs, depending on one's point of view, this is not necessarily the case. For example, from the point of view of every student in an IVET-based classroom, the instructor can be looking directly at each one of them. 
Hence, the sum of mutual gazes in the natural environment cannot exceed $100 \%$ across all possible pairs of instructor-learner dyads. However, in IVEs, it can be $100 \%$ for every possible dyad.

In an exploratory study (Bailenson et al. 2005), we tested the hypothesis that audience members as a group would agree more with a persuader under conditions of non-zero sum gaze than under more "natural" conditions in an IVE. (Details regarding the technology can be found in the cited reference immediately above.) In this study we ran males and females in a collaborative IVE under one of three gaze conditions: natural, augmented, and reduced (see Fig. 3). Three same sex people, a presenter and two listeners, occupied the IVE and could see each other's avatars by slightly moving their heads in all conditions. The latter were naïve participants randomly assigned to condition. The presenter was specially trained for purposes of this study.

In the natural condition, the presenter's actual physical movements were rendered via his or her avatar. In the augmented condition, the presenter's avatar gazed directly at each listener $100 \%$ of the time, while still exhibiting slight animations in head movements so as not to appear static or "frozen." In the reduced condition, the presenter's avatar gazed down at his virtual computer monitor $100 \%$ of the time.

The results of this study were mixed. As predicted, females were reliably more persuaded during the augmented (i.e., non-zero sum) condition than in the natural and reduced gaze conditions. Men did not show this effect, though the lack of differences is consistent with sex differences in the utilization of nonverbal cues overall and gaze in particular (e.g., Mulac et al. 1987). Interestingly, even though participants in the augmented gaze condition estimated upon recall that the presenter looked at them a higher proportion (slightly greater than 50\%) of the time than participants in the other conditions, they apparently were unaware that the presenter looked at them $100 \%$ of the time. These latter data suggest that demand characteristics were not operating to produce the study's results.

\section{Situational context TSI: ideal seat in an IVE}

In traditional classrooms, student seating plans tend to either be dictated by instructor's rule (e.g., alphabetical; alternating rows of males and females) or by student's choice (e.g., preferences for front, back, or middle seating). One might surmise that students would choose their ideal seat with the goal of better classroom learning and performance, but there may be competing motives (e.g., staying out of the instructor's typical gaze pattern, sitting with friends). In either case, the degrees of freedom for seat choice decrease with the number of seats already chosen or assigned. In an IVE classroom, however, there is no such constraint. Every student can sit in an ideal seat that would facilitate learning and performance.

We conducted a study (reported as study 3 in Bailenson et al. 2008) in which participants were students in an IVE classroom (see Fig. 4). Seat location was manipulated

Fig. 3 Design of nonzero sum gaze study

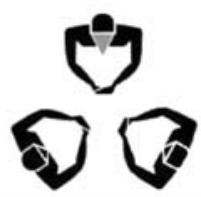

Reduced

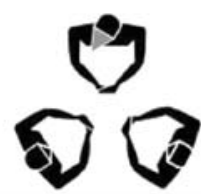

Natural

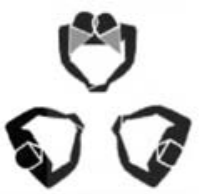

Augmented 

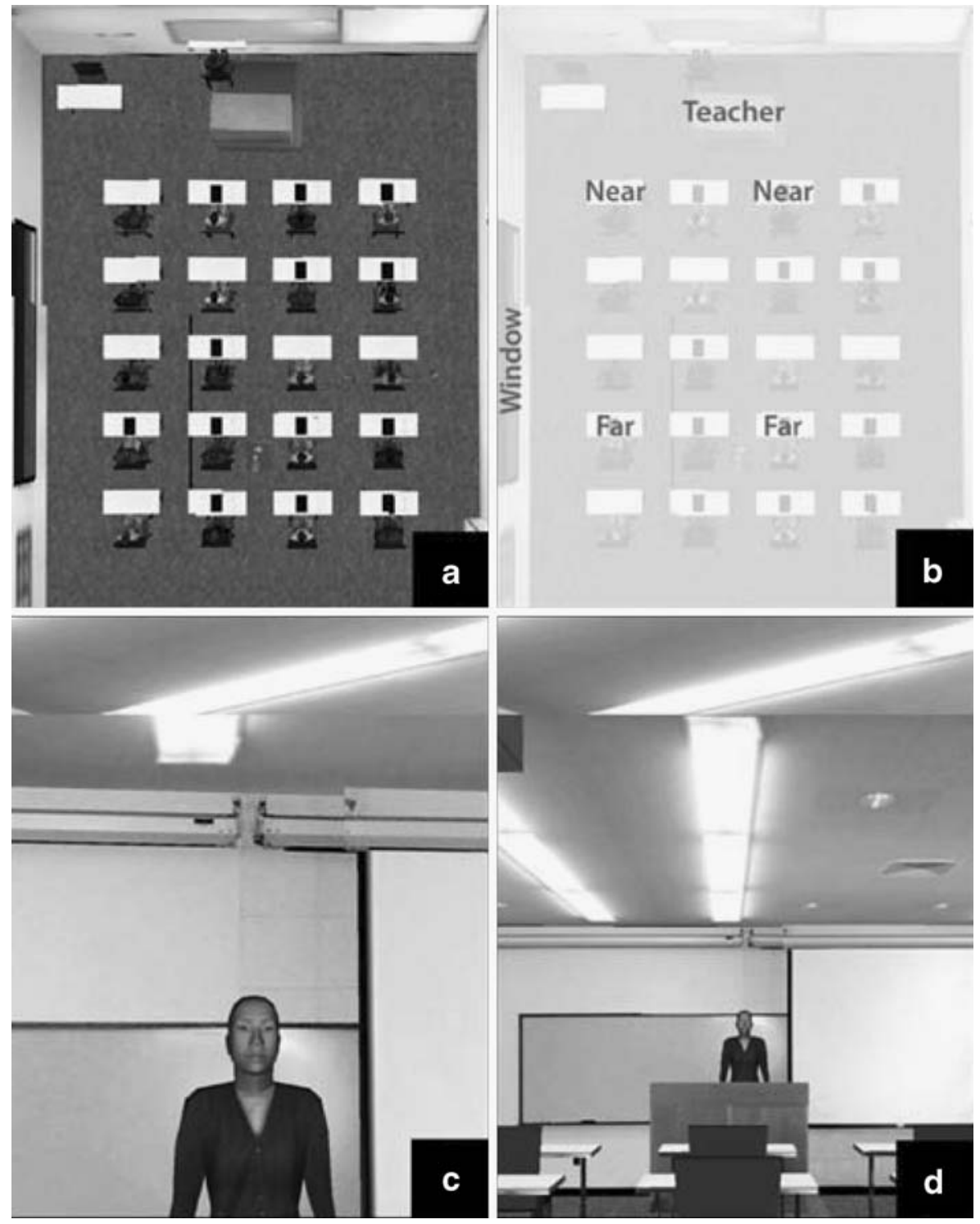

Fig. 4 "Student" locations in ideal seat study

within subjects such that participants sat in four different virtual seats (two, $2.5 \mathrm{~m}$ from the instructor and two $8.5 \mathrm{~m}$ from the instructor). Number of students in the classroom was varied between subjects such that all seats in the IVE classroom were filled with "students" (i.e., idling agents) or the IVE classroom was devoid of other students. The teacher via an avatar delivered an eight minute learning passage in four 2-min segments. During each segment students were digitally moved to one of the four seats comprising the within subjects manipulation in an order counterbalanced across participants. Participants completed a test comprised of an equal number of questions relevant to each learning/ seating segment. 
The results of this study demonstrated significantly better performance on tests for segments delivered when students sat in closer proximity to the instructor's avatar. Further, the results revealed a heightened proximity effect on test performance when they sat closer to the instructor immediately after having sat farther away.

Sensory ability TSI: monitoring one's gaze

One reason that classroom proxemics are important may be that instructors do not spread their gaze evenly across students. Any student learning problems stemming from lack of instructor gaze (e.g., lack of attention, mind wandering) can be exacerbated via the motivation on the part of some students to avoid instructor gaze. In another study (reported as Study 1 in Bailenson et al. 2008), we investigated the effects of a specific sensory ability TSI; namely, information fed back to instructors regarding the distribution of their gaze across students in an IVE classroom. We predicted that instructors with such information would spread their gaze more uniformly than instructors without the information.

Study participants played the role of instructor in an IVE classroom comprised of nine students (i.e., virtual agents) who exhibited pre-recorded head movements appropriate to instructor-student classroom interaction. The two critical variables that were manipulated were student location and TSI (gaze feedback information) availability. In the critical conditions, participant instructors received feedback or did not while delivering an 8-min lecture. In the feedback condition, "instructors" received real-time information about his or her own gaze behavior by the opacity of the digital student agents' appearance. More specifically, the opacity of a student agent was a function of the instructor's gaze such that the student agent would become increasingly transparent over time with lack of instructor gaze.

Gaze inattention, or the amount of time that the participant instructor avoided gazing at student agents, was the major dependent variable. As Fig. 5 depicts, the results revealed

Fig. 5 Inattention data from extrasensory feedback study

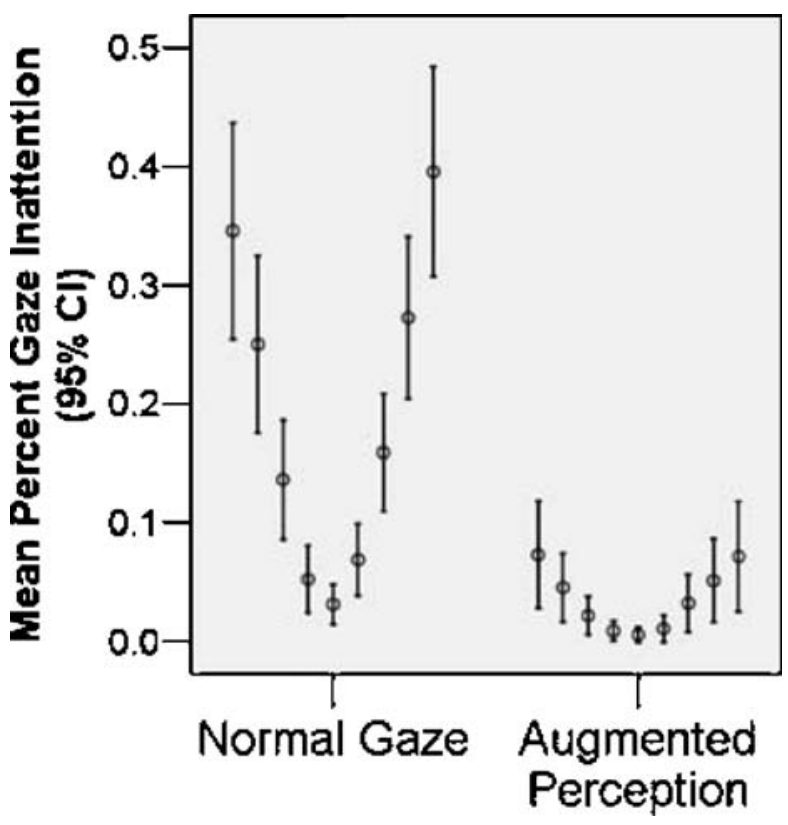


significant main effects for feedback condition and student seating location and a significant interaction such that inattention decreased the most for students seated in the periphery.

\section{Implications for Adaptivity}

The three studies reviewed above provide examples of how IVET produced TSIs can affect instructional and learning processes in IVE-based didactic contexts. Although the three studies differ in terms of specific types of TSIs (i.e., self-presentational, contextual, and sensory), they all involve visual attention in some way. This was not meant to exclude other types (e.g., social facilitation/inhibition types, auditory types) of which there are many. However, they do illustrate the power of IVET for enabling instructors and student to perform their roles better.

In terms of the theoretical model of social influence within immersive virtual environments, the independent variables in these studies varied along the dimension of communicative realism; more specifically, movement realism (i.e., mutual gaze and proxemics) of somewhat anthropometrically realistic digital human representations. Interestingly, the results of the studies reported here and elsewhere (e.g., Bailenson et al. 2008) suggest that theory of mind (i.e., whether one or more of the IVE interactants are known to be agents or avatars) is relatively unimportant for these and similar TSIs in, at least, instructional settings. This comports with the theoretical model in the sense that TSIgenerated effects can occur at the automatic or unconscious level in participants.

Of course, these studies and others are not without limitations. They represent only short instructional interactions among people playing the social roles of students and instructors. In addition, the results (i.e., nonzero sum effects for females but not for males in the first study reviewed) suggest that group and individual differences in the targets of IVE-based instructional TSIs need investigation.

In terms of adaptivity, digital IVET-based systems can be designed to be adaptive and, therefore, responsive to group and individual differences. Different instructor TSIs can be designed and employed for different groups of learners as well as different individual learners. At a more general level, instructor agents or avatars, for example, can be rendered as the same race, gender, socioeconomic class, etc. as learners. At a more micro-level, for example, the system can learn those instructor agent/avatar movements that cause particular learners to better attend to the instructor (e.g., via learner head and eye movement) or to their emotional state (e.g., via facial pattern recognition) and the system can train itself to render the instructor optimally. The adaptive power of digital IVET-based learning systems is practically limitless.

Commentary-Andreas Harrer This paper presents an overview of the authors' theory and research exploring the potential and characteristics of immersive virtual environments for education. This is highly relevant because of the widespread acceptance of virtual environments as venues for social interaction, be it for social networking, such as in Second Life, for gaming as in massive multiplayer games, for education, etc. Interestingly, educational usage of digital environments still mainly takes a conservative stance, preserving the educational practice of nondigital environments as far as possible.

Gerhard Fischer (1998) maintains that such preservation involves technology merely as porting existing educational practices into computer systems (e.g., using and providing digital slides in a web space as an extension of overhead projection) and has been criticized there for its lack of innovation. Fischer posed challenged educators to create new frameworks of learning based on the capabilities of new systems. In virtual environments, constraints of physical education settings, such as teacher attention toward individual students, physical location of seats etc. can be coped with differently than in nondigital classrooms. 
The authors provide evidence that augmenting teachers' gaze so that she/he attends to every virtual student can improve her/his persuasive power. Similarly, the authors demonstrated that learning was increased when students sat virtually near the instructor's avatar, a situation that can be induced easily in a virtual environment.

Yet, learner characteristics have to be taken into account, too: in the gaze augmentation study, the effect was significant for women but not for men. This makes the challenge for creation of digital learning environments a twofold one: on the one hand, the factors influencing student attention and cognition have to be carefully explored, such as in the presented studies; on the other hand, the results of the studies have to be integrated in practical educational environments that are adaptive towards the learners' traits, i.e., augmentations should only be used for the populations, where the effect of the instrument benefits learning. These adaptive educational systems are currently most advanced when considering knowledge levels of students (this field is traditionally called Intelligent Tutoring Systems) (Woolf 2008), but a broader consideration of motivational, social, attentional, and other aspects is necessary to move "beyond giftwrapping technology," thus allowing new learning approaches supported by digital, virtual, and immersive environments.

Open Access This article is distributed under the terms of the Creative Commons Attribution Noncommercial License which permits any noncommercial use, distribution, and reproduction in any medium, provided the original author(s) and source are credited.

\section{References}

Bailenson, J. N. (2006). Transformed social interaction in collaborative virtual environments. In P. Messaris \& L. Humphreys (Eds.), Digital media: Transformations in human communication (pp. 255-264). New York: Peter Lang.

Bailenson, J., \& Blascovich, J. (2005). Avatars. In W. S. Bainbridge \& William Sims (Eds.), Berkshire encyclopedia of human-computer interaction. Great Barrington: Berkshire.

Bailenson, J. N., \& Beall, A. C. (2006). Transformed social interaction: Exploring the digital plasticity of avatars. In R. Schroeder \& A. Axelsson (Eds.), Avatars at work and play: Collaboration and interaction in shared virtual environments (pp. 1-16). New York: Springer.

Bailenson, J., Blascovich, J., Beall, A. C., \& Loomis, J. M. (2001). Proxemic behavior as a function of behavioral realism within immersive virtual environments. Presence: Teleoperators and Virtual Environments, 12, 183-196.

Bailenson, J. N., Beall, A. C., Blascovich, J., Loomis, J., \& Turk, M. (2005). Transformed social interaction, augmented gaze, and social influence in immersive virtual environments. Human Communication Research, 31, 511-537.

Bailenson, J. N., Yee, N., Blascovich, J., Beall, A. C., Lundblad, N., \& Jin, M. (2008). The use of immersive virtual reality in the learning sciences: Digital transformations of teachers, students, and social context. The Journal of the Learning Sciences, 17, 102-141.

Blascovich, J., Loomis, J., Beall, A., Swinth, K., Hoyt, C., \& Bailenson, J. (2002). Immersive virtual environment technology as a research tool for social psychology. Psychological Inquiry, 13, 103125.

Burgoon, J. K., Dunbar, N. E., \& Segrin, C. (2002). Nonverbal influence. In J. P. Dillard \& M. Pfau (Eds.), The persuasion handbook: Developments in theory and practice (pp. 445-473). Thousand Oaks: Sage.

Fischer, G. (1998). Making learning a part of life-beyond the 'gift-wrapping' approach of technology. In P. Alheit \& E. Kammler (Eds.), Lifelong learning and its impact on social and regional development (pp. 435-462). Bremen: Donat.

Garau, M., SlaterM, P. D., \& Razzeque, S. (2005). The responses of people to virtual humans in an immersive virtual environments. Presence, 14, 104-116.

Goffman, E. (1961). Asylums: Essays on the social situation of mental patients and other inmates. New York: Doubleday.

Graesser, A. C., Millis, K. K., \& Zwann, R. A. (1997). Annual Review of Psychology, 48, 163-189.

Heider, F., \& Simmel, M. (1944). An experimental study of apparent behavior. American Journal of Psychology, 57, 243-259.

Mulac, A., Studley, L. B., Weimann, J. M., \& Bradac, J. J. (1987). Male-female gaze in same sex and mixed sex dyads: Gender-linked differences in mutual influence. Human Communication Research, 13, 323343 . 
Premack, D. G., \& Woodruff, G. (1978). Does the chimpanzee have a theory of mind? Behavioral and Brain Sciences, 1, 515-526.

Roussou, M., Oliver, M., \& Slater, M. (2008). Exploring activity theory as a tool for evaluating interactivity and learning in virtual environments for children. Cognition, Technology \& Work, 10, 141-153.

Uleman, J., \& Bargh, J. (1989). Unintended thought. New York: Guilford.

Wheatley, T., Milleville, S. C., \& Martin, A. (2007). Understanding animate agents: Distinct roles for the social network and mirror system. Psychological Science, 18, 469-474.

Woolf, B. P. (2008). Building intelligent interactive tutors. Amsterdam: Elsevier. 